ARTICLE

Received 14 Oct 2013 | Accepted 12 May 2014 | Published 9 Jun $2014 \quad$ DOl: 10.1038/ncomms5091

\title{
A histone H3K36 chromatin switch coordinates DNA double-strand break repair pathway choice
}

Chen-Chun Pai ${ }^{1, \star}$, Rachel S. Deegan ${ }^{1}{ }^{*}$, Lakxmi Subramanian${ }^{2}$, Csenge Gal $^{3}$, Sovan Sarkar ${ }^{1}$, Elizabeth J. Blaikley ${ }^{1}$, Carol Walker ${ }^{1}$, Lydia Hulme ${ }^{1}$, Eric Bernhard ${ }^{1}$, Sandra Codlin ${ }^{4}$, Jürg Bähler ${ }^{4}$, Robin Allshire ${ }^{2}$, Simon Whitehall ${ }^{3}$ \& Timothy C. Humphrey ${ }^{1}$

DNA double-strand break (DSB) repair is a highly regulated process performed predominantly by non-homologous end joining (NHEJ) or homologous recombination (HR) pathways. How these pathways are coordinated in the context of chromatin is unclear. Here we uncover a role for histone H3K36 modification in regulating DSB repair pathway choice in fission yeast. We find Set2-dependent H3K36 methylation reduces chromatin accessibility, reduces resection and promotes NHEJ, while antagonistic Gcn5-dependent H3K36 acetylation increases chromatin accessibility, increases resection and promotes $H R$. Accordingly, loss of Set2 increases H3K36Ac, chromatin accessibility and resection, while Gcn5 loss results in the opposite phenotypes following DSB induction. Further, H3K36 modification is cell cycle regulated with Set2-dependent H3K36 methylation peaking in G1 when NHEJ occurs, while Gcn5-dependent H3K36 acetylation peaks in S/G2 when HR prevails. These findings support an $\mathrm{H} 3 \mathrm{~K} 36$ chromatin switch in regulating DSB repair pathway choice.

\footnotetext{
${ }^{1}$ CRUK MRC Oxford Institute for Radiation Oncology, Department of Oncology, University of Oxford, ORCRB, Roosevelt Drive, Oxford OX3 7DQ, UK. ${ }^{2}$ Wellcome Trust Centre for Cell Biology, Institute of Cell Biology, The University of Edinburgh, Swann Building, Mayfield Road, Edinburgh EH9 3JR, UK.

${ }^{3}$ Institute for Cell and Molecular Biosciences, The Medical School, Newcastle University, Framlington Place, Newcastle upon Tyne, NE2 4HH, UK. ${ }^{4}$ University College London, Department of Genetics, Evolution and Environment, Darwin Building, Gower Street, London WC1E 6BT, UK. * These authors contributed equally to this work. Correspondence and requests for materials should be addressed to T.C.H. (email: timothy.humphrey@oncology.ox.ac.uk).
} 
$\mathrm{D}$ NA double-strand breaks (DSBs) if unrepaired or inappropriately repaired can lead to cell death or genomic instability ${ }^{1}$. To prevent such undesirable outcomes, cells employ the evolutionarily conserved non-homologous end joining (NHEJ) or homologous recombination (HR) repair pathways to restore genome integrity. During NHEJ, the broken ends are protected by the Ku70/80 heterodimer, which in mammalian cells facilitates recruitment of the DNA-dependent protein kinase (DNA-PKcs). This facilitates processing of damaged DNA ends and subsequent ligation of the compatible ends through the activity of the conserved DNA ligase 4, XLF, XRCC4 complex ${ }^{2}$. HR is initiated by resection of the $5^{\prime}$ end of the DSB to generate a $3^{\prime}$ single-stranded DNA (ssDNA) overhang. This is bound by replication protein A (RPA), and, during mitotic recombination, a Rad51 nucleofilament is formed promoting strand invasion of the sister chromatid or homologous chromosome, which is used as a repair template before second end capture ${ }^{3}$. DSB repair pathway choice is influenced by a number of factors including cell cycle phase. In yeast, NHEJ is restricted to G1, while HR operates in S and G2 phase cells when a sister chromatid is available as a repair template ${ }^{4-6}$. DSB

a

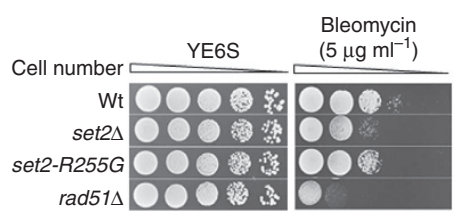

C

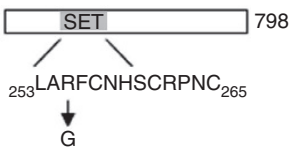

d

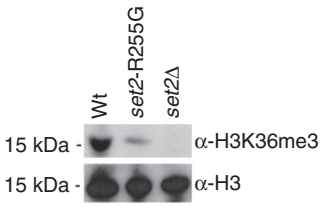

b

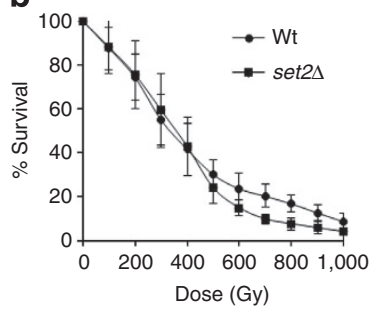

e

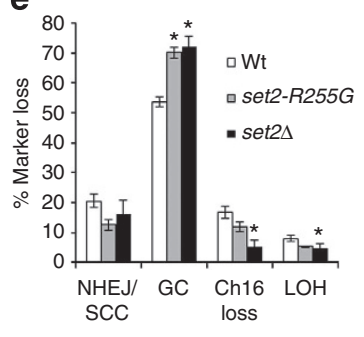

f

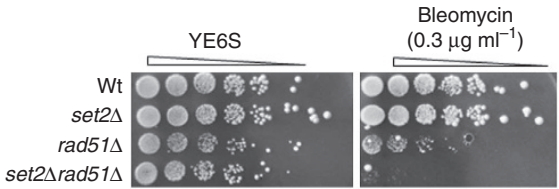

Figure 1 | Set2 is required to suppress HR and for resistance to DNA-damaging agents. (a) 10-fold serial dilutions of wild-type, set2 4 , set2-R255G and rad51D strains on YE6S, YE6S $+5 \mu \mathrm{g} / \mathrm{ml}$ bleomycin. Plates were incubated at $32^{\circ} \mathrm{C}$ for 3 days. At least two biological replicates were performed. (b) IR survival curve for wild-type (Wt) and set $2 \Delta$ strains. Means \pm s.e. of three experiments are shown. (c) A schematic of the structure of Set2 with SET (ASW) domain shown with amino-acid sequences indicating arginine residue mutated in SET domain of the set2R255G mutant. (d) Western blot analysis of H3K36me3 levels in wild-type $(\mathrm{Wt})$, set2 $\Delta$ and set2-R255G cells. (e) Percentage DSB-induced marker loss in wild type (Wt), set2 $\Delta$ and set2- $R 255 \mathrm{G}$ containing $\mathrm{Ch}^{16}-\mathrm{RMGAH}$. The levels of NHEJ/SCC, GC, Ch ${ }^{16}$ loss and LOH are shown. Means \pm s.e. of three experiments are shown. ${ }^{*}$ represents significant difference compared with wild-type $(P<0.05, t$-test). See also Supplementary Fig. 1 and Table 1. (f) $\operatorname{set} 2 \Delta$ cells are sensitive to bleomycin in combination with rad51 . Fivefold serial dilutions of wild-type $(\mathrm{Wt})$, set2 $\Delta$, rad51 $\Delta$ and $\operatorname{set} 2 \Delta \mathrm{rad} 51 \Delta$ cells were grown on YE6S and YE6S $+0.3 \mu \mathrm{g} / \mathrm{ml}^{-1}$ bleomycin. resection is a critical determinant of repair pathway choice and is highly regulated as inappropriate pathway deployment can result in pathological consequences ${ }^{1}$.

Here we have investigated the role of histone H3 lysine 36 (H3K36) modification in DSB repair pathway choice. H3K36 methylation is associated with numerous functions ${ }^{7}$. In Saccharomyces cerevisiae, SET (Su(var)3-9, Ez, Trithorax) domain-containing 2 (Set2) is responsible for mono, di and trimethylation of H3K36 (ref. 8). In humans, H3K36 methylation is catalysed through the activities of eight distinct enzymes, while SETD2/HYPB uniquely catalyses the trimethylation of $\mathrm{H} 3 \mathrm{~K} 36$ (ref. 9). Importantly, SETD2 has recently been classified as a novel tumour suppressor, suggesting a role in genome stability ${ }^{10-14}$ Links between histone H3K36 methylation and DSB repair have been identified in yeast and human cells ${ }^{15-17}$. These findings support a role for $\mathrm{H} 3 \mathrm{~K} 36$ methylation in promoting efficient NHEJ, although the molecular basis of this is unknown. A role for H3K36 methylation in promoting $\mathrm{HR}$ has also been recently described $^{18}$. Histone $\mathrm{H} 3 \mathrm{~K} 36$ residues can also be acetylated, which in $S$. cerevisiae is performed by the Gon5 histone acetyltransferase $(\mathrm{HAT})^{19}$. Gcn5 is the catalytic subunit of the SAGA, ADA and SLIK chromatin-modifying complexes that post-translationally modify histones and regulate gene expression $^{20}$. Gcn5 has also been associated with DSB repair in yeast and human cells, and these data suggest a possible role in $\mathrm{HR}^{21-24}$. Structurally, H3K36 residues can be either methylated or acetylated raising the intriguing possibility that these exclusive marks might drive distinct biological effects within chromatin ${ }^{19}$. As NHEJ and HR pathways can exhibit an antagonistic relationship ${ }^{25,26}$, we have investigated the functional interplay between these $\mathrm{H} 3 \mathrm{~K} 36$ modifications in regulating DSB repair pathway choice in fission yeast.

Here we identify a role for Set2-dependent H3K36 methylation in facilitating NHEJ. In contrast, we find Gcn5-dependent H3K36 acetylation promotes HR. Together our findings support a role for an H3K36 chromatin switch in coordinating DSB repair pathway choice in fission yeast.

\section{Results}

Set2 methyltransferase suppresses homologous recombination. To determine a possible role for $\mathrm{H} 3 \mathrm{~K} 36$ modification in DSB repair, we examined the effect of deleting Set2 on damage sensitivity. set $2 \Delta$ cells was found to exhibit modest sensitivity to both the radiomimetic bleomycin and ionizing radiation (IR) compared with wild-type cells, indicating a role for Set2 in the cellular response to DSBs (Fig. 1a,b). To investigate the role of Set2 methyltransferase activity in the DNA damage response, a highly conserved arginine within the catalytic SET domain was mutated to glycine $(\operatorname{set} 2-R 255 G)$ within the endogenous set ${ }^{+}$ gene, which was predicted to disrupt the methyltransferase activity (Fig. 1c) ${ }^{8,27}$. No H3K36me3 was observed in set $2 \Delta$ cells, indicating an absolute requirement for Set2 in H3K36 trimethylation in fission yeast, as previously described ${ }^{28}$ (Fig. 1d). Substantially reduced levels of H3K36me3 were observed in the set2-R255G mutant compared with wild type, indicating that this residue is required for optimal H3K36 methylation (Fig. 1d). To investigate a possible role for Set2 in DSB repair, we used a DSB assay to quantitate marker loss profiles and thus repair responses to a site-specific DSB within a non-essential minichromosome ${ }^{29}$ (Supplementary Fig. 1). This revealed that deletion of set $2^{+}$resulted in significantly elevated levels of gene conversion (GC) $(72 \% P=0.02)$, compared with wild type (55\%) (Fig. 1e and Table 1). HO induction in a set2-R255G background resulted in a very similar DSB repair profile to set $2 \Delta$ with significantly elevated levels of GC 
Table 1 | Analysis of DSB repair outcomes in different genetic backgrounds.

\begin{tabular}{|c|c|c|c|c|c|c|c|c|}
\hline Genetic background & $\%$ NHEJ/SCC & $P$-value & $\%$ GC & P-value & $\% \mathrm{Ch}^{16}$ loss & P-value & $\%$ LOH & $P$-value \\
\hline Wild type $\left(\mathrm{Ch}^{16}-\mathrm{RMGAH}\right)$ & $20 \pm 2.3$ & - & $55 \pm 2.3$ & - & $17 \pm 1.4$ & - & $7 \pm 1.0$ & - \\
\hline $\operatorname{set} 2 \Delta\left(\mathrm{Ch}^{16}-\mathrm{RMGAH}\right)$ & $16 \pm 4.6$ & 0.57 & $72 \pm 3.4$ & 0.02 & $5 \pm 2.2$ & 0.02 & $5 \pm 1.5$ & 0.09 \\
\hline set2-R255G ( $\left.\mathrm{Ch}^{16}-\mathrm{RMGAH}\right)$ & $12 \pm 1.8$ & 0.24 & $70 \pm 1.8$ & $<0.01$ & $12 \pm 1.7$ & 0.10 & $5 \pm 0.1$ & $<0.01$ \\
\hline$g c n 5 \Delta\left(\mathrm{Ch}^{16}-\mathrm{RMYAH}\right)$ & $33 \pm 1.6$ & $<0.01$ & $45 \pm 3.0$ & $<0.01$ & $14 \pm 1.5$ & 0.18 & $6 \pm 0.1$ & 0.04 \\
\hline gcn5 $\operatorname{lig} 4 \Delta\left(\mathrm{Ch}^{16}-\mathrm{RMYAH}\right)$ & $23 \pm 1.5$ & $<0.01$ & $56 \pm 1.5$ & 0.02 & $14 \pm 2.2$ & 0.07 & $6 \pm<0.01$ & 0.03 \\
\hline $\operatorname{set} 2 \Delta \operatorname{gcn} 5 \Delta\left(\mathrm{Ch}^{16}-\mathrm{RMYAH}\right)$ & $21 \pm 1.4$ & $<0.01$ & $71 \pm 1.7$ & 0.65 & $5 \pm 0.2$ & 0.01 & $2 \pm 0.3$ & $<0.01$ \\
\hline
\end{tabular}

For each genetic background the assay was repeated three times (with independent isolates), such that $>1,000$ colonies were scored. Mean \pm s.e. of the three experiments are shown. A single blank vector control was also analysed in each genetic background to give a spontaneous level of $\mathrm{Ch}^{16}$ loss, which was subtracted to calculate the break-induced values shown above. $P$-values ( $t$-test) are against wild type $\mathrm{Ch}^{16}$-RMYAH unless otherwise stated.

(70\% $P<0.01$ ), compared with wild type (Fig. 1e and Table 1), thus identifying a role for Set 2 methyltransferase activity in suppressing HR repair. Consistent with an HR-independent role for Set 2 in DSB repair, a double mutant $\operatorname{set} 2 \Delta$ rad51 $\Delta$ exhibited acute sensitivity to bleomycin compared with the single mutants (Fig. 1f), indicating that Set2 is required for survival in the absence of HR.

Set2 methyltransferase is required for canonical NHEJ. As HR and NHEJ can compete during DSB repair ${ }^{25,26}$, the increased HR observed in the set $2 \Delta$ and set2-R255G backgrounds could have arisen from reduced NHEJ, which may have been masked by sister chromatid conversion (SCC) in our DSB assay (Supplementary Fig. 1). Consistent with a role for Set2 in $\mathrm{NHEJ}, \operatorname{lig} 4 \Delta$ was found to be epistatic with $\operatorname{set} 2 \Delta$ in response to bleomycin (Fig. 2a). Further, quantitating colony survival indicated that the set $2 \Delta$ lig $4 \Delta$ double mutant phenocopied the sensitivity of $\operatorname{set} 2 \Delta$ to bleocin (Fig. $2 b$ ). These data support a role for Set 2 in canonical NHEJ. However, as set $2 \Delta$ was more sensitive than lig $4 \Delta$, Set2 must also perform an additional NHEJindependent function in response to DNA damage. The ability of Set2 to repair a DSB by NHEJ was further assessed using a plasmid-rejoining assay, in which recircularization of linearized LEU2 plasmids by NHEJ allows stable propagation of leu ${ }^{+}$ colonies. LEU2 plasmids linearized with PstI ( $3^{\prime}$ overhang), EcoRI ( $5^{\prime}$ overhang) or PvuII (blunt) were transformed into wild-type, lig $4 \Delta$, and set $2 \Delta$ cells and the number of leu ${ }^{+}$colonies quantified. Plasmid rejoining was impaired in set $2 \Delta$ cells compared with wild-type cells (Fig. 2c). Plasmid rejoining was also impaired in the set2-R255G strain (Supplementary Fig. 2). These results together define a role for Set 2 methyltransferase activity in promoting canonical NHEJ and are consistent with impaired NHEJ in set $2 \Delta$ cells leading to increased HR. No significant changes in gene expression of NHEJ or HR repair genes were observed in set $2 \Delta$ cells in the absence or presence of damage, suggesting a direct role in NHEJ. (Supplementary Fig. 3a,b).

To determine the mechanism by which Set 2 promotes NHEJ, we investigated whether $\mathrm{H} 3 \mathrm{~K} 36 \mathrm{me}$-interacting proteins (readers) functioned in NHEJ. In S. cerevisiae, co-transcriptional methylation of H3K36 by Set2 leads to recruitment of the Rpd3S HDAC complex, which deacetylates histones in the wake of elongating PolII ${ }^{30}$. However, analysis of alp $13 \Delta$ or $\operatorname{clr} 6-1$, which disrupt the equivalent deacetylase complex in $S$. pombe $e^{31,32}$, failed to disrupt NHEJ (Supplementary Fig. 4a,b), indicating that Set 2 functions independently of the Clr6 HDAC complex to promote NHEJ. To further investigate the role of Set 2 in promoting NHEJ and suppressing $\mathrm{HR}$, we considered a possible role for Set2 in protecting DSB ends from resection. To test this, levels of RPA foci were investigated following exposure to 50 Gy IR using a construct in which the large subunit of RPA was GFP-tagged

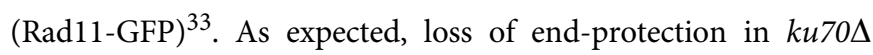
cells leads to increased Rad11-GFP foci following DSB induction $^{34}$ (Supplementary Fig. 5). Similarly, an increase in Rad11-GFP foci was observed in a set2-R255G mutant background compared with wild type (Fig. 2d), suggesting a role for Set2 methyltransferase activity in preventing breakinduced ssDNA formation analogously to $\mathrm{Ku} 70$, thus providing an explanation for the increase in HR in the absence of Set2. We also observed increased level of RPA levels in G1-arrested set $2 \Delta$ cells compared with G1-arrested wild-type cells (Supplementary Fig. 6).

We next investigated whether H3K36 methylation was induced in response to a site-specific DSB. Chromatin immunoprecipitation (ChIP) analysis revealed that $\mathrm{H} 3 \mathrm{~K} 36 \mathrm{me} 3$ levels increase in a Set2-dependent manner following $\mathrm{HO}$-induced DSB induction (Fig. 2e). Given the loss of end protection and increased HR observed following Set2 loss, we examined a possible role for Set2-dependent H3K36 methylation in Ku recruitment to a DSB. ChIP analysis revealed a significant reduction in the levels of Ku80-myc associated with a HO-induced DSB in a set $2 \Delta$ background (Fig. 2f). Together these findings support a role for Set2-dependent $\mathrm{H} 3 \mathrm{~K} 36$ methylation in $\mathrm{Ku}$ recruitment to breaksites thereby facilitating NHEJ.

H3K36 methylation and acetylation are mutually inhibitory. Lysine residues in proteins can be methylated or acetylated in a mutually exclusive manner. As loss of Set2 led to DSB end deprotection and increased HR, we investigated whether these events were associated with loss of $\mathrm{H} 3 \mathrm{~K} 36$ methylation and increased $\mathrm{H} 3 \mathrm{~K} 36$ acetylation. In budding yeast, H3K36 is acetylated by the Gcn 5 histone acetyltransferase (HAT) ${ }^{19}$. To examine the relationship between H3K36 modifications, levels of H3K36 methylation and acetylation were analysed from nuclear extracts of wild-type, set $2 \Delta$ or $g c n 5 \Delta$ cells by western blot analysis. In contrast to wild type, $\mathrm{H} 3 \mathrm{~K} 36 \mathrm{ac}$ was undetectable in $g c n 5 \Delta$ cells, thus defining an evolutionarily conserved role for Gcn 5 as the H3K36 HAT in fission yeast, as in budding yeast (Fig. 3a) ${ }^{19}$. In the absence of Set2, H3K36ac levels were elevated compared with wild type (Fig. 3a). As expected, no H3K36me3 was observed in set $2 \Delta$ cells, consistent with Set2 being essential for H3K36 methylation (Figs 1d and 3b). Surprisingly, H3K36me3 levels in $\operatorname{gcn} 5 \Delta$ cells were much higher than that observed in wildtype cells (Fig. 3b). Thus, Gcn5-dependent H3K36ac inhibits $\mathrm{H} 3 \mathrm{~K} 36 \mathrm{me} 3$, indicating that these $\mathrm{H} 3 \mathrm{~K} 36$ modifications are mutually inhibitory.

Gcn 5 promotes HR and suppresses NHEJ. As Gcn5-dependent H3K36ac inhibits Set2-dependent H3K36me, which our data indicate is required for NHEJ, we investigated whether Gcn5 facilitated HR repair. We found that $g c n 5 \Delta$ cells exhibited mild sensitivity to bleomycin (Fig. 3c) consistent with a role for Gcn5 
a

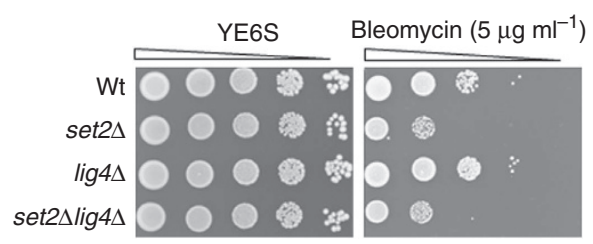

C

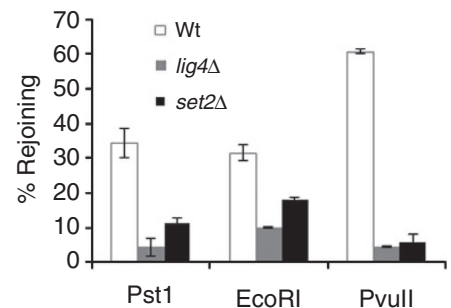

e

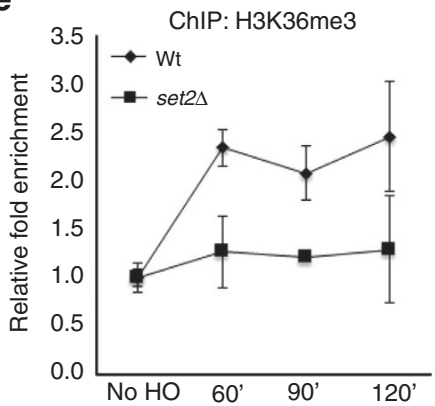

b

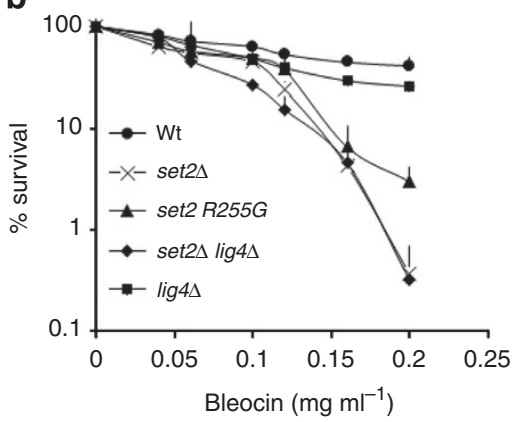

d

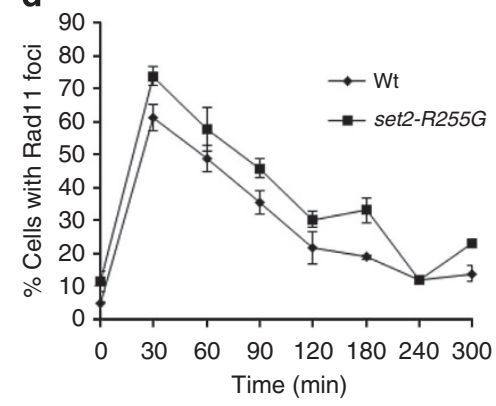

f

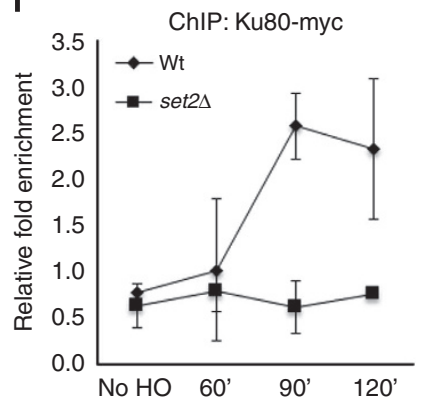

Figure 2 | Set2 is required for C-NHEJ. (a) Serial dilutions of the indicated strains were spotted onto YE6S containing $5 \mu \mathrm{ml} \mathrm{ml}^{-1}$ bleomycin or no drug. Plates were incubated at $32^{\circ} \mathrm{C}$ for 3 days. (b) Percentage survival of strains indicated compared with wild-type following plating on YE6S $+/-$ the indicated bleocin concentration. Plates were incubated for 3 days at $32{ }^{\circ} \mathrm{C}$ and then scored. Means \pm s.e. of four independent experiments are shown. (c) Set2 is required for NHEJ plasmid rejoining. The percentage of leu ${ }^{+}$colonies obtained following transformation rejoining of a LEU2 plasmid (pAL19) linearized with EcoRI, Pstl or Pvull following transformation into wild-type (Wt), lig $4 \Delta$ and set2 $\Delta$ strains compared with uncut plasmid control is shown. Means \pm s.e. of three experiments are shown. See also Supplementary Fig. 2. (d) Quantification of Rpa1(Rad11)-GFP foci in wild-type (Wt) and set2-R255G strains following exposure to 50 Gy IR treatment. Mean \pm s.e. of three experiments are shown. See also Supplementary Fig. 5. (e) DSB induction results in a Set2-dependent increase in histone H3K36me3 levels proximal to the HO break. qChIP enrichments of H3K36me3 in wild-type (Wt) or set2 $\Delta$ cells at various times (60, 90 or $120 \mathrm{~min}$ ) following urg-HO induced DSB induction at the MATa site between SPAC3H1.10 and hsr1 on Chromosome I, as previously described $^{59}$. Enrichment of immunoprecipitated DNA at $50 \mathrm{bp}$ from the break relative to that at act is presented, as a ratio of that observed in a strain expressing no $\mathrm{HO}$ endonuclease. Error bars represent s.d. from at least two biological replicates. (f) DSB induction results in a Set2-dependent increase in Ku80 levels proximal to the $\mathrm{HO}$ break. qChIP enrichments of Ku80-myc in wild-type (Wt) or set2 $2 \Delta$ cells at various times (60, 90 or 120 min) following urg- $\mathrm{HO}$ induction as described above. Enrichment of immunoprecipitated DNA at $50 \mathrm{bp}$ from the break, relative to that at the fbp 1 locus is presented. Error bars represent s.d. from at least two biological replicates.

in DSB repair ${ }^{35}$. Microarray analysis previously performed on gcn5 $\Delta$ cells did not identify any alteration in the transcription of DSB repair genes ${ }^{36}$, suggesting a direct role for Gcn5 in DSB repair. Therefore, a role for Gen5 in DSB repair was further examined using the DSB assay (Supplementary Fig. 1). DSB induction in a $g c n 5 \Delta$ background resulted in significantly increased levels of NHEJ/SCC $(33 \%, P<0.01)$ and significantly reduced GC $(45 \%, P<0.01)$ compared with wild-type cells (Fig. $3 \mathrm{~d}$ and Table 1). These results identified a role for Gcn5 in promoting efficient HR. Further, these results contrasted with those observed following loss of Set2 methyltransferase.

To investigate when Gcn5 acts during HR, the kinetics of Rad51-CFP foci were analysed in $g c n 5 \Delta$ cells following treatment with IR. $g c n 5 \Delta$ cells exhibited a striking reduction in Rad51-CFP foci 30-60 min following treatment with 50 Gy IR, compared with wild-type cells (Fig. 3e). Following this initial decline, the percentage of cells with Rad51-CFP foci increased again $120 \mathrm{~min}$ after damage. Importantly, Rad51-CFP expression was not affected in gcn5 5 cells (Supplementary Fig. 7). These data indicated that $g c n 5 \Delta$ cells, although still able to recruit Rad51, did so much less effectively than wild-type cells, indicating a role for Gcn5 in HR before strand invasion (synapsis). To further address the presynaptic role of Gcn5, the recruitment of RPA subunit Rad11-GFP to ssDNA following exposure to 50 Gy IR was examined. In contrast to wild-type cells, the levels of Rad11-GFP foci were reduced in $g c n 5 \Delta$ cells at earlier time points (Fig. 3f). Therefore, Gcn5 functions presynaptically during HR to promote ssDNA formation following DSB induction. We noted that again, 
a

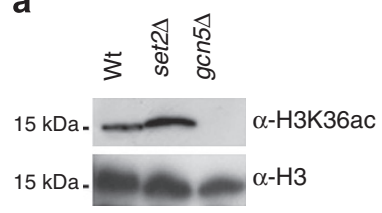

b

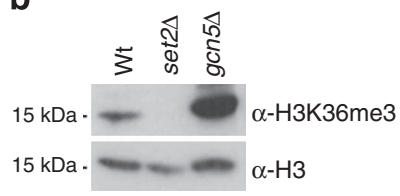

C

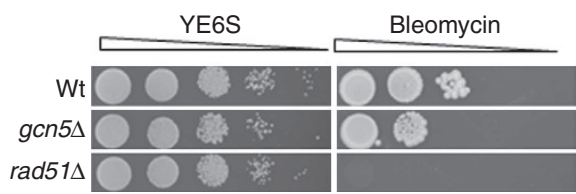

e

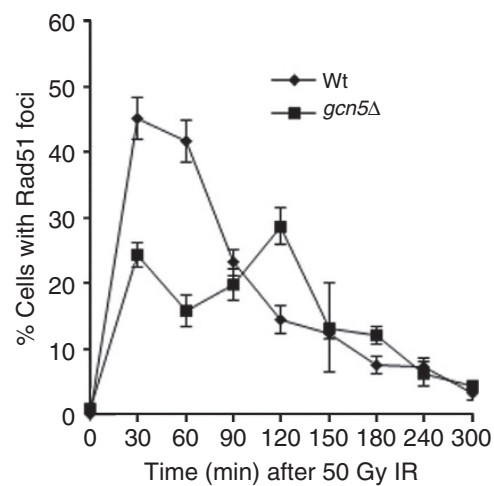

$\mathbf{f}$

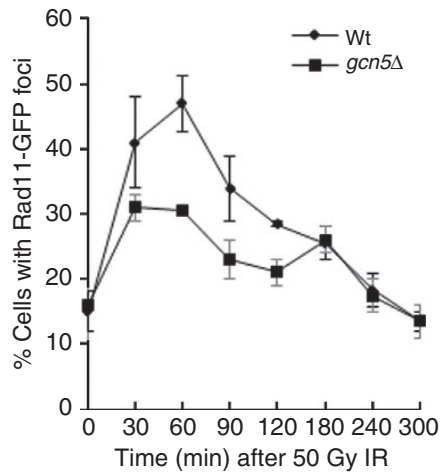

d

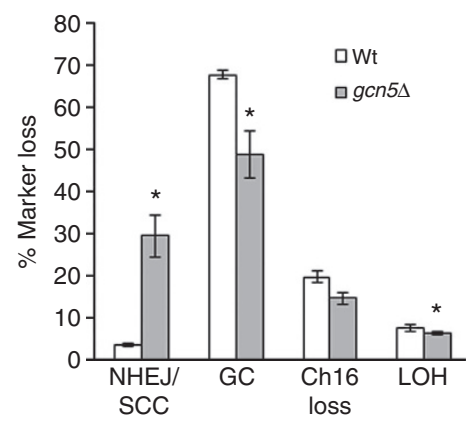

g

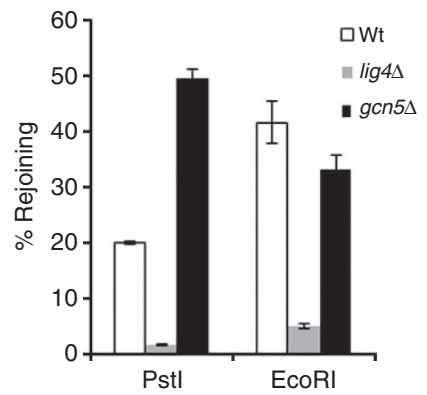

$\mathbf{h}$

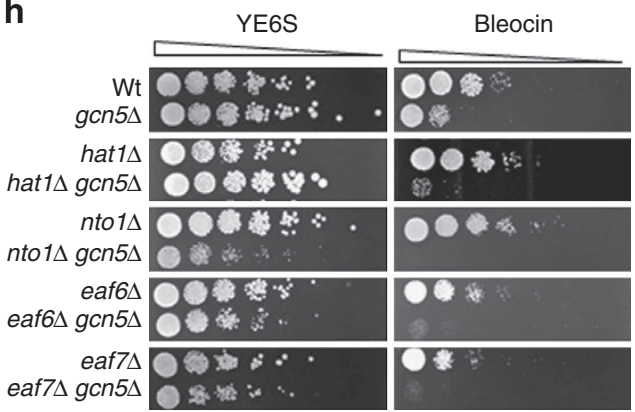

Figure 3 | Gcn5 promotes HR and suppresses NHEJ. (a,b) Methylation and acetylation of H3K36 are mutually inhibitory. (a) Western blot analysis of H3K36me3 in wild type (Wt), set2 $\Delta$ and gcn5 $\Delta$ nuclear extract. (b) Western blot analysis of H3K36ac in wild type (Wt), set2 $\Delta$ and gcn5 $\Delta$ nuclear extract.

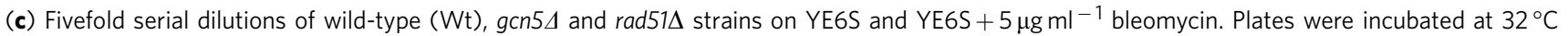
for 3 days. At least two biological replicates were performed. (d) Percentage DSB-induced marker loss in wild type (Wt) and gcn5 $\Delta$ containing $\mathrm{Ch}^{16}$-RMYAH. The levels of NHEJ/SCC, GC, $\mathrm{Ch}^{16}$ loss and $\mathrm{LOH}$ are shown. Means \pm s.e. of three experiments are shown. ${ }^{*}$ represents significant difference compared with wild type ( $P<0.05, t$-test). See also Table 1. (e) Quantification of Rad51-CFP foci in wild-type and gcn5 $\Delta$ strains following exposure to 50 Gy IR. (f) Quantification of Rpa1(Rad11)-GFP foci in wild-type and gcn5 $\Delta$ strains strains following exposure to 50 Gy IR treatment. Data are the mean of three experiments and error bars ( \pm s.e.) are shown. (g) Gcn5 suppresses NHEJ plasmid rejoining. The percentage of leu ${ }^{+}$colonies obtained following transformation and rejoining of a LEU2 plasmid (pAL19) linearized with EcoRI, Pstl into wild-type, lig $4 \Delta$ and gcn5 $\Delta$ strains compared with uncut plasmid control is shown. Means \pm s.e. of three experiments are shown. (h) Gcn5 functions redundantly with other HAT complex subunits in the DNA damage response. Fivefold serial dilutions of wild-type (Wt), gcn5 $\Delta$, hat $1 \Delta$, hat $1 \Delta$ gcn5 $\Delta$, nto $1 \Delta$, nto $1 \Delta$ gcn5 $\Delta$, eaf6 $\Delta$, eaf $6 \Delta$ gcn5 $\Delta$, eaf $7 \Delta$, and eaf7 $\operatorname{gcn} 5 \Delta$ strains on YE6S and YE6S $+5 \mu \mathrm{gl}^{-1}$ bleocin. At least two biological replicates were performed.

Gcn5 loss exhibited an opposite phenotype to that observed in a set2-R255G background (Fig. 2d).

Given the reduced ssDNA formation following DSB induction, we further addressed whether DSB repair was associated with elevated levels of NHEJ in a $g c n 5 \Delta$ background. To confirm whether NHEJ was increased following DSB induction in the $g c n 5 \Delta$ mutant (Fig. 3d), repair in a $g c n 5 \Delta$ lig $4 \Delta$ double mutant was assessed using the DSB assay (Supplementary Fig. 1). The proportion of NHEJ/SCC colonies was reduced in a gcn5 $\Delta$ lig $4 \Delta$ background (23\%) compared with $g c n 5 \Delta$ (33\%) consistent with
$10 \%$ of this population being attributable to NHEJ in a $g c n 5 \Delta$ strain (Table 1). This level is significantly greater than the NHEJ/ SCC level in wild-type cells $(4 \% P<0.01)$, and thus represents a significant increase in NHEJ in the gcn $5 \Delta$ mutant. A similar profile was observed in a $g c n 5 \Delta \operatorname{set} 2 \Delta$ double mutant (Table 1 ). Further analysis by the plasmid-rejoining assay indicated that $g c n 5 \Delta$ cells exhibited consistently increased rejoining of a PstI linearized plasmid compared to wild-type cells, although this appeared to depend on the presence of a $3^{\prime}$ overhang, as the same was not observed for an EcoRI-linearized plasmid (Fig. 3g). 
As $g c n 5 \Delta$ exhibited mild sensitivity to DNA damage compared with $\operatorname{rad} 51 \Delta$ (Fig. 3c), we tested whether Gcn5 functioned redundantly with other HAT complexes to facilitate the DNA damage response. We found that deletion of $g c n 5^{+}$together with

a

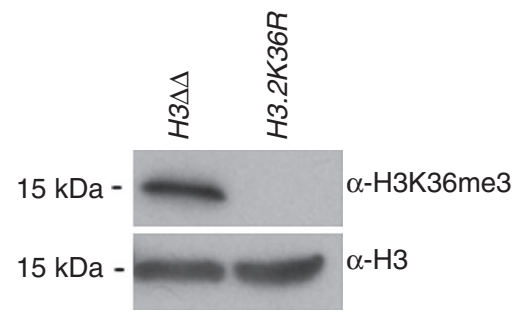

b

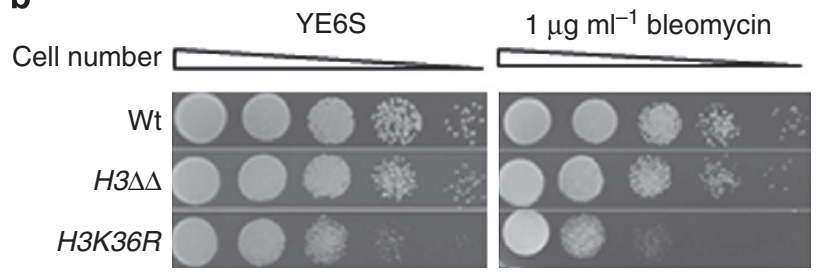

C

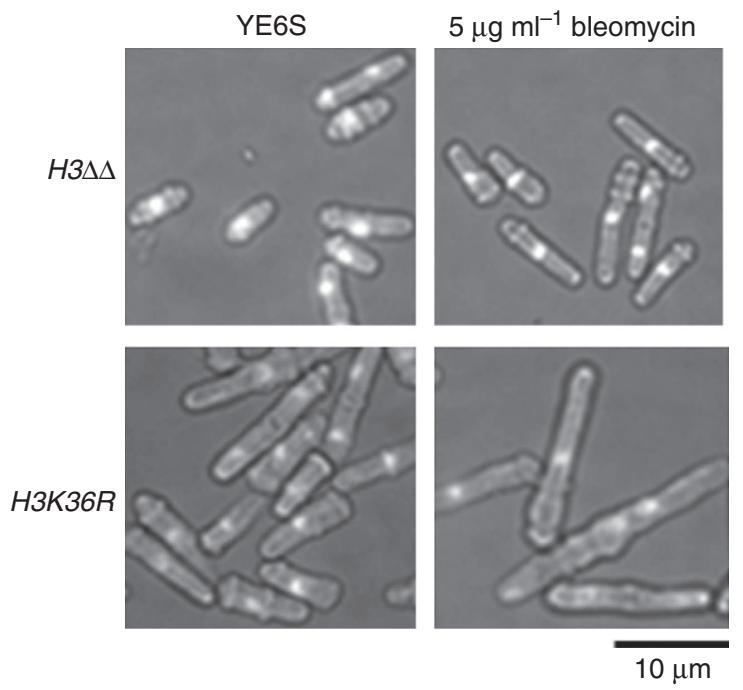

d

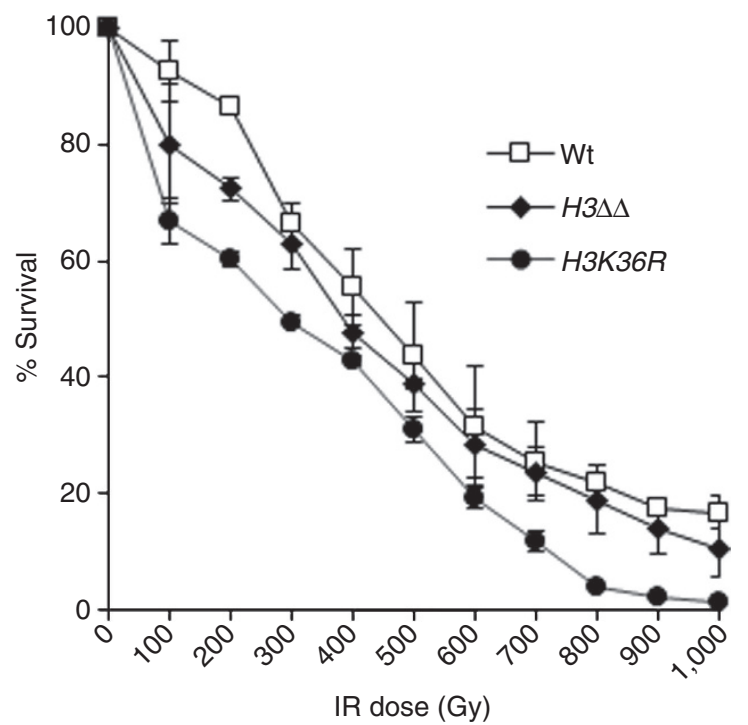

genes encoding the histone acetyltransferase Hatl (ref. 37), Nto1 (a subunit of the NuA3/Mst2 complex) ${ }^{35}$, Eaf6 (a subunit of both $\mathrm{NuA} 3$ and $\mathrm{NuA} 4)^{35}$ or Eaf7 (a subunit of the NuA4 complex) ${ }^{38}$ resulted in a striking increase in bleocin sensitivity compared with the single mutants (Fig. 3h). These findings support a key role for Gcn5 in facilitating the DNA damage response in conjunction with other HAT complexes. These findings are consistent with and extend previous observations ${ }^{35,37,39}$.

Role for H3K36 modification in DSB repair. To test the role of $\mathrm{H} 3 \mathrm{~K} 36$ modification in DSB repair, we tested the sensitivity of an $H 3 K 36 R$ mutant to DSB-inducing agents. No H3K36me3 was detected in $H 3 K 36 R$ cells (Fig. $4 \mathrm{a}$ ). The H3K36R mutant exhibited increased sensitivity to bleomycin (Fig. $4 \mathrm{~b}$ ) compared with wildtype or control cells $(H 3.1 \Delta H 3.3 \Delta)$ that retained an intact copy of the $H 3.2$ gene. $H 3 K 36 \mathrm{R}$ mutant cells were elongated after $6 \mathrm{~h}$ exposure to bleomycin, in contrast to wild-type cells, consistent with a checkpoint-dependent cell cycle delay resulting from failed DSB repair (Fig. 4c). Further, the $H 3 K 36 R$ mutant exhibited increased IR sensitivity compared with wild-type or $H 3.1 \Delta H 3.3 \Delta$ controls (Fig. 4d). In addition, the H3K36R mutation did not affect total histone H3 levels (Supplementary Fig. 8). These findings are in accordance with a role for $\mathrm{H} 3 \mathrm{~K} 36$ modification in DSB repair.

H3K36 modification and chromatin accessibility. To address how H3K36 modification might affect DSB repair, we probed chromatin accessibility. Lysine acetylation neutralizes the positive charge on histones and thus weakens interactions between histones and DNA. Thus, more open chromatin arising from lysine acetylation may facilitate resection and subsequent DSB repair by HR. Conversely, lysine methylation might compact chromatin thereby inhibiting resection and promoting NHEJ. We therefore tested the global effect of deleting set $2^{+}$or $g c n 5^{+}$on chromatin accessibility to micrococcal nuclease (MNase) following exposure to bleocin, as previously described ${ }^{35}$. We used the percentage of DNA that has a low molecular weight DNA ( $<$ tetranucleosome, $\sim 600 \mathrm{bp}$ ) as a measure of chromatin accessibility. Deleting set ${ }^{+}$ resulted in an increase in the level of low molecular weight DNA fragments, consistent with increased chromatin accessibility following DNA damage. In contrast, deleting $g c n 5^{+}$resulted in reduced levels of MNase fragments of $600 \mathrm{bp}$ or less, consistent with reduced chromatin accessibility in response to DNA damage (Fig. 5a-c). No obvious difference in DNA accessibility was observed in wild-type, set $2 \Delta$ or $g c n 5 \Delta$ backgrounds in the absence of DNA damage (Fig. 5d). These findings are consistent with Set2 and Gen5 regulating DSB repair pathway choice through modulating H3K36 methylation/acetylation status and subsequently chromatin accessibility to DSB repair factors.

H3K36 modification is cell cycle regulated. In fission yeast, DSB repair pathway choice is cell cycle regulated, with NHEJ being utilized during G1, while HR is employed in S and G2 phases ${ }^{6}$.

Figure 4 | Histone H3K36 is required for survival following exposure to DSB-inducing agents. (a) Western blot analysis of $\mathrm{H} 3 \mathrm{~K} 36 \mathrm{me} 3$ in wild-type $(\mathrm{Wt})$ and $H 3.2 K 36 R \mathrm{H} 3.1 \Delta H 3.3 \Delta(H 3 K 36 R)$ cells. $\alpha-H 3$ is shown as a loading

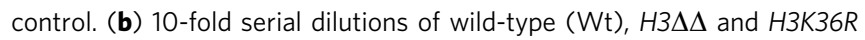
cells on YE6S, YE6S $+1 \mu \mathrm{g} \mathrm{ml}^{-1}$ bleomycin. Plates were incubated at $32^{\circ} \mathrm{C}$ for 3 days. (c) Methanol-fixed asynchronous wild-type (Wt) and H3K36R cells imaged following growth in the presence or absence of $5 \mu \mathrm{g} \mathrm{ml} \mathrm{l}^{-1}$ bleomycin for $6 \mathrm{~h}$. Bar, $10 \mu \mathrm{m}$. (d) IR survival curve for wild-type (Wt) and H3K36R cells. Data are the mean of three experiments and error bars ( \pm s.e.) are shown. 
a

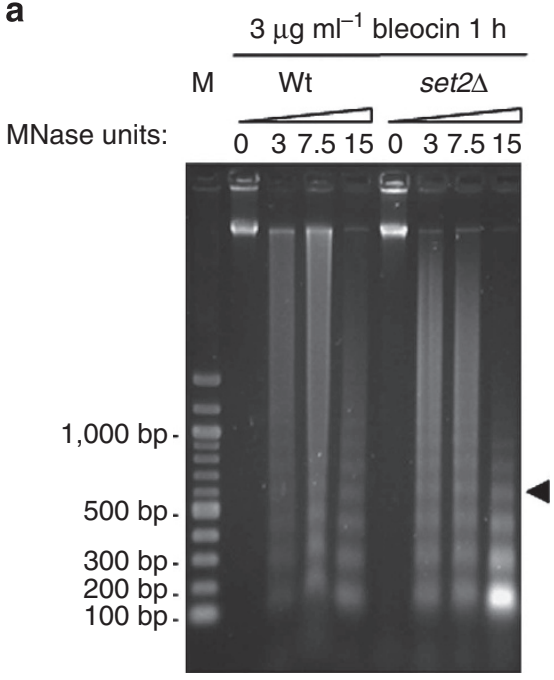

b

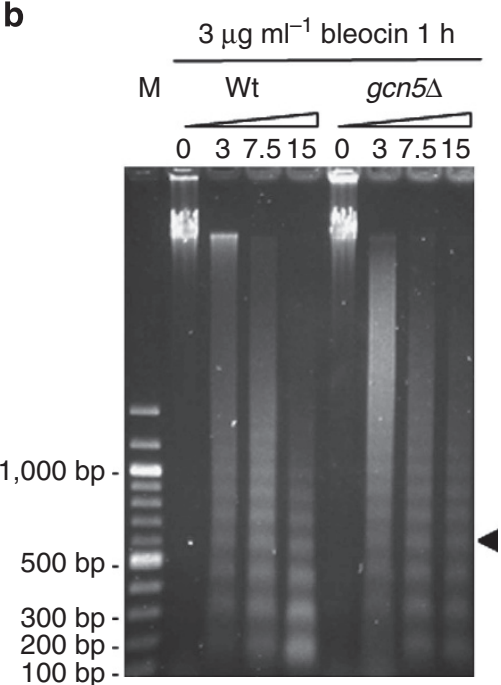

C

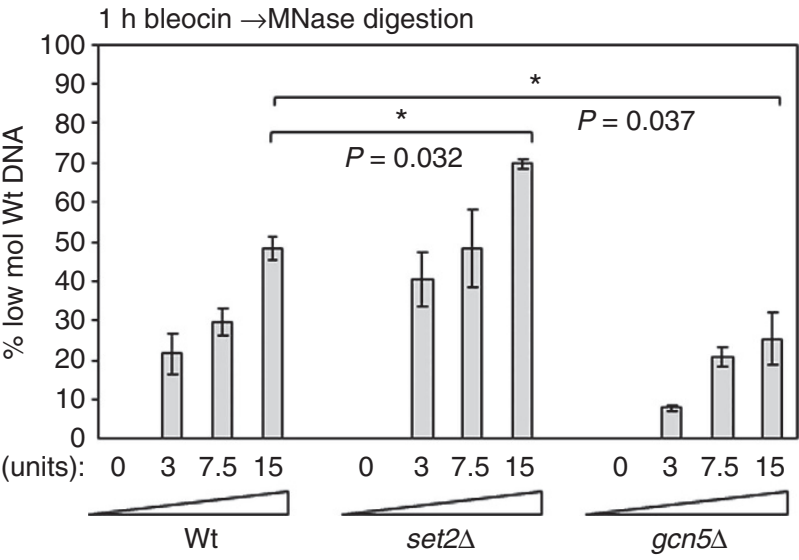

d

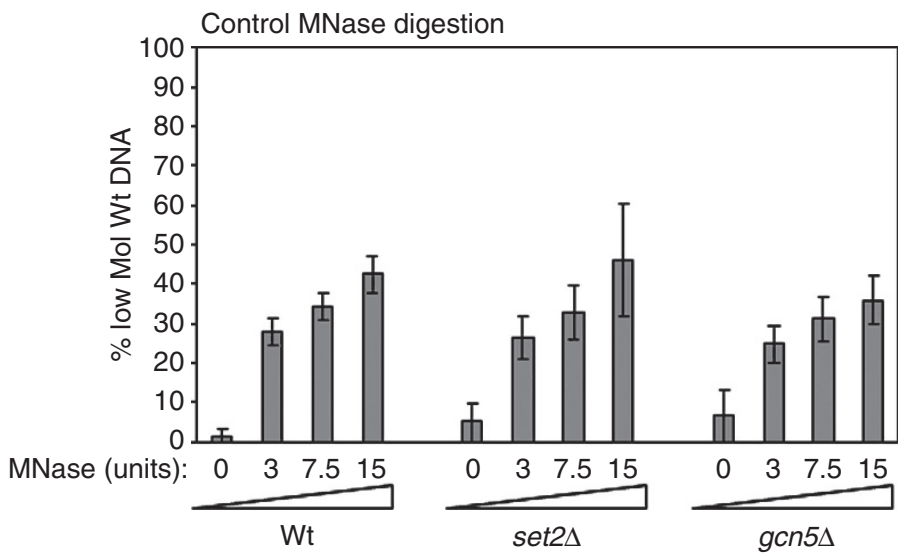

Figure 5 | Chromatin is more accessible to MNase following DNA damage in set2 $\Delta$ cells and less accessible in gcn5 $\Delta$ cells. (a,b) Mid-log phase cells were incubated in $3 \mu \mathrm{g} \mathrm{ml}^{-1}$ bleocin for $1 \mathrm{~h}$ followed by MNase digestion for $10 \mathrm{~min}$ at the indicated concentrations. Digested chromatin DNA was resolved by gel electrophoresis and detected by ethidium bromide staining. The gels are representative of three independent repeats. (c) MNase digested chromatin DNA resolved on agarose gels were analyzed using Image J. The proportion of low molecular weight DNA (<tetranucleosome) was calculated as a percentage of the total sample. set $2 \Delta$ cells have a significantly higher proportion of low molecular weight particles following digestion with $15 \mathrm{U}$ MNase $(P=0.032$; $t$-test), while gcn5 $\Delta$ cells have a significantly lower population of low molecular weight particles when compared with wild type $(P=0.037$; $t$-test), indicating an increase in set $2 \Delta$ and a decrease in $g c n 5 \Delta$ chromatin accessibility. Data are the mean of at least three independent repeats and error bars ( \pm s.e.) are shown ( ${ }^{\star}$ denotes $P<0.05$; $t$-test). (d) Control MNase digestions (no bleocin treatment) were analysed as described for $\mathbf{c}$. Data are the mean of at least three independent repeats and error bars $( \pm$ s.e.) are shown. Comparison of set $2 \Delta$ and gcn5 $\Delta$ samples with wild-type revealed no significant change in the proportion of low molecular weight particles ( $P$-value cutoff 0.05 ; $t$-test). 
a

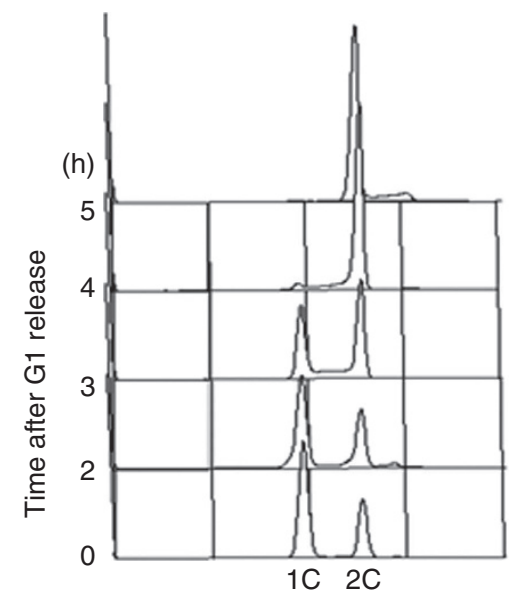

b

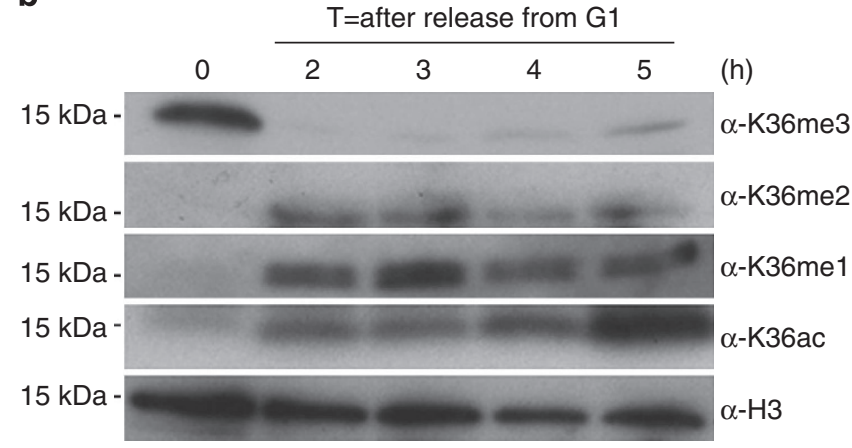

Figure 6 | H3K36 methylation and acetylation are cell cycle regulated.

(a) Flow cytometry analysis of wild-type cells following release from nitrogen starvation. The data shown were performed from at least two biological replicates. (b) Acid-extracted histones prepared from a wild-type strain were resolved on a 4-20\% SDS-PAGE gel, transferred to a PVDF membrane and probed for H3K36me3, H3K36me1 and H3K36ac. $\alpha-\mathrm{H} 3$ is shown as a loading control. Western blot analysis of H3K36me3,

$\mathrm{H} 3 \mathrm{~K} 36 \mathrm{me2}, \mathrm{H} 3 \mathrm{~K} 36 \mathrm{me} 1$ and H3K36Ac in wild-type nuclear extract at the times shown following release from nitrogen starvation.

This prompted us to investigate whether $\mathrm{H} 3 \mathrm{~K} 36$ modification was cell cycle regulated. Following arrest in G1 by nitrogen starvation and release into the cell cycle (Fig. 6a), levels of chromatin bound H3K36 methylation and acetylation were determined by western blot. Cells with an increased 1C DNA content exhibited strikingly high H3K36me3 levels, while H3K36ac levels were low (Fig. 6a,b). Cell cycle progression resulted in a significant reduction in $\mathrm{H} 3 \mathrm{~K} 36 \mathrm{me} 3$ levels after $2 \mathrm{~h}$, whereas levels of H3K36me2 and H3K36me1 increased, consistent with S-phase progression (Fig. 6a). H3K36ac levels also increased with progression through S-phase, peaking at $5 \mathrm{~h}$ when the majority of cells were in G2 (Fig. 6b). These results indicate that modification of the H3K36 residue is cell cycle regulated. Further, the peak of methylation in a 1C population and acetylation in S/G2 is consistent with Set2-dependent H3K36 methylation being required for NHEJ, and Gcn5-dependent H3K36 acetylation being required for HR. The protein levels of Set2-myc and Gcn5-myc are constant during the cell cycle (Supplementary Fig. 9).

\section{Discussion}

Our findings support an H3K36 modification-mediated switch in coordinating DSB repair pathway choice in fission yeast. We define roles for Set2-dependent H3K36 methylation in reducing chromatin accessibility, reducing DSB resection and promoting NHEJ through $\mathrm{Ku}$ recruitment. In contrast, Gcn5-dependent H3K36 acetylation increases chromatin accessibility, increases DSB resection and promotes HR. Accordingly, loss of Set2 results in increased Gcn5-dependent H3K36Ac, open chromatin, increased resection and increased HR, while loss of Gcn5 results in increased Set2-dependent H3K36me, closed chromatin, reduced resection and increased NHEJ. The role for H3K36 modification in coordinating DSB repair was further confirmed by the observation that $H 3 K 36 R$ mutation was sensitive to bleomycin and IR. Moreover, we found H3K36 modification to be cell cycle regulated with chromatin-bound $\mathrm{H} 3 \mathrm{~K} 36 \mathrm{me} 3$ peaking in G1 where NHEJ occurs while H3K36 acetylation peaked in S/G2 phase when HR predominates. Together these findings support an $\mathrm{H} 3 \mathrm{~K} 36$ chromatin switch in coordinating DSB repair pathway choice in fission yeast.

How might a switch in chromatin states dictated by H3K36 coordinate DSB repair pathway choice? Here we consider two non-exclusive models suggested by these and other findings. In the first 'recruitment' model, Set2-dependent H3K36 methylation is required to recruit the Ku70-Ku80 heterodimer to the breaksite thereby promoting NHEJ. In contrast, Gen5-dependent H3K36 acetylation may be refractory to $\mathrm{Ku}$ recruitment and could instead function to recruit HR factors. In this respect, Set2 may promote $\mathrm{Ku}$ recruitment through potential readers of the H3K36me mark. However, we found that neither Clr6, the Rpd3 homologue, nor Alp13, a subunit of the Clr6 HDAC complex II, exhibited defects in NHEJ. Instead, alp13 $\Delta$ and the temperaturesensitive clr6-1 allele were acutely sensitive to bleomycin ${ }^{31,32}$, and the set $2 \Delta$ alp $13 \Delta$ double mutant exhibited increased sensitivity, thus indicating a distinct function for the HDAC complex II in the DSB response. Other currently unknown H3K36me readers may promote $\mathrm{Ku}$ recruitment. Alternatively, H3K36me may recruit $\mathrm{Ku}$ indirectly through other chromatin factors. In this respect, components of the RSC chromatin remodelling complex physically interact with $\mathrm{Ku} 80$, and RSC has previously been shown to be required for loading of $\mathrm{Ku}$ onto breaks ${ }^{40,41}$. However, it is possible that $\mathrm{Ku}$, which has a strong affinity for duplex DNA ends in vitro ${ }^{42}$, binds DNA ends independently of chromatin or associated factors in vivo.

In a second 'chromatin accessibility' model, the distinct H3K36 chromatin states control end resection at a break-site via chromatin accessibility. Here, Set2-dependent H3K36me is proposed to promote closed chromatin, protecting ends from resection, thereby facilitating $\mathrm{Ku}$ recruitment or retention, and NHEJ. In contrast, Gcn5-dependent H3K36Ac is proposed to promote open acetylated chromatin and/or increased histone exchange, thus facilitating resection, reducing $\mathrm{Ku}$ binding or recruitment, and thus increasing HR. Consistent with this model, Set2 has been shown to suppress histone $\mathrm{H} 3$ and $\mathrm{H} 4$ acetylation by preventing histone exchange during transcription in S. cerevisiae. Increased histone exchange in the absence of Set2 facilitates histone acetylation thereby leading to increased cryptic transcripts ${ }^{43,44}$. In the context of DSB repair, increased histone exchange may facilitate transient nucleosome removal or the dynamic incorporation of histone variants thereby facilitating resection at DSBs. Indeed, a recent study using budding yeast characterizing the impact of chromatin on in vitro resection found that efficient resection by Sgs1-Dna2 required nucleosomefree regions adjacent to the DSB, while resection by Exo1 was completely blocked by nucleosomes. Moreover, incorporation of the histone variant H2A.Z was found to enhance resection ${ }^{45}$. Thus, Set2 may promote NHEJ through reducing chromatin accessibility to the resection machinery. The increased levels of Gcn5-dependent H3K36ac and damage-induced nucleosome mobility in a set $2 \Delta$ background, together with increased ssDNA 


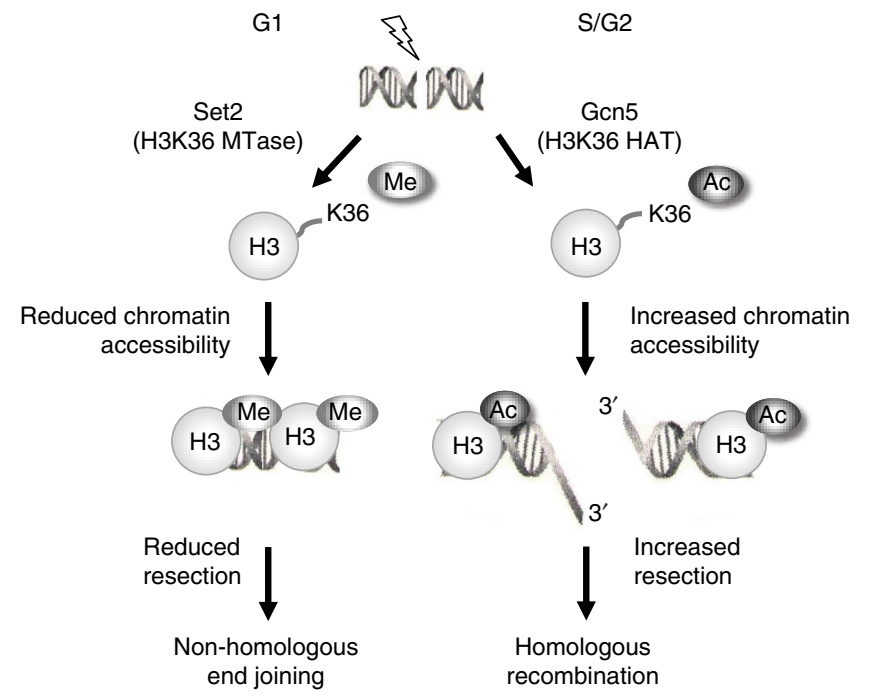

Figure 7 | An H3K36 chromatin switch regulates DSB repair pathway choice in fission yeast. In G1 high levels of Set2-dependent H3K36me (indicated) reduces nucleosome accessibility to repair factors, resulting in reduced resection and increased NHEJ repair of DSBs. In S/G2 high levels of Gcn5-dependent H3K36Ac (indicated) increases nucleosome accessibility to repair factors, resulting in increased resection and increased HR repair of DSBs. See text for details.

formation following IR leading to increased HR compared with wild-type, are consistent with such a model (Fig. 7).

Our findings support an early role for Gcn5-dependent H3K36Ac in facilitating HR. Loss of Gcn5 alone exhibited a modest sensitivity to DNA damaging agents; moreover, both Rad11 and Rad51 foci formation were delayed rather than abrogated in the absence of Gcn5. However, Gcn5 was found to play a critical role in the DNA damage response in conjunction with other HATs, including Hat1, NuA3 and NuA4, which have been previously associated with the DNA damage response and DSB repair ${ }^{35,39,46}$. Recently, recruitment of chromatin remodelling enzymes to a DSB was found to be dependent on the early steps of HR, while inhibited by NHEJ in budding yeast ${ }^{47}$. We speculate that Gcn5 may function early in HR in conjunction with other HATs to promote recruitment of ATP-dependent remodelers, repair factors and/or to increase DNA accessibility, thus promoting resection and HR.

Importantly, H3K36 methylation is associated with DSB repair in other organisms. Indeed, roles for Set2-dependent H3K36 methylation in transcription-coupled DNA damage checkpoint activation in $S$. cerevisiae are reported in an accompanying manuscript ${ }^{48}$. Moreover, a H3K36 modification switch may help coordinate DSB repair pathway choice in humans. H3K36 dimethylation was reported to be increased at DSBs and to enhance NHEJ through recruiting NBS1 and Ku70 repair factors in a METNASE-dependent manner ${ }^{17}$. In contrast, a role for SETD2-dependent H3K36 trimethylation in facilitating HR in actively transcribed regions has recently been described ${ }^{49,50}$. Here, SETD2-dependent H3K36 trimethylation promotes HR through constitutive recruitment of Lens Epithelial Growth Factor p75 (LEDGF) to chromatin. In response to DNA damage, LEDGF recruits C-terminal binding protein interacting protein (CtIP), which promotes resection, thereby facilitating HR repair ${ }^{18}$. However, while LEDGF is present in higher eukaryotes, it is not evolutionarily conserved in budding or fission yeasts. Interestingly, Set1-dependent H3K4 methylation at DSBs also promotes NHEJ through $\mathrm{Ku}$ recruitment in budding yeast ${ }^{51}$.
Thus, histone methylation plays an important role in facilitating NHEJ. How these methylation events are coordinated, and their functional antagonism through demethylation and acetylation is of considerable interest.

The cell cycle regulation of $\mathrm{H} 3 \mathrm{~K} 36$ modification is surprising given the wide range of functions associated with H3K36 methylation. However, this finding is consistent with observations recently reported in human cells ${ }^{52}$ and indicates that $\mathrm{H} 3 \mathrm{~K} 36$ cell cycle modification is evolutionarily conserved. How H3K36 modification is coordinated through the cell cycle and the functional implications for such regulation are currently unknown. The interplay between H3K36 acetylation and methylation has previously been proposed to regulate gene expression ${ }^{19}$. Here we show that this H3K36 chromatin switch helps to control DSB repair pathway choice in fission yeast. This chromatin switch, in concert with the complex networks that control DSB repair, ensures that the fidelity of the genome is maintained, preventing deleterious chromosomal rearrangements as a consequence of misuse of repair mechanisms.

\section{Methods}

Yeast strains, media and genetic methods. The strains used in this study are listed in Supplementary Tables 1 and 2. Standard media and growth conditions were used throughout this work ${ }^{53}$. Cultures were grown in rich media (YE5S) or minimal media (EMM) at $32{ }^{\circ} \mathrm{C}$ with shaking, unless otherwise stated.

Site-specific DSB assay. Cells were grown exponentially in liquid culture for $48 \mathrm{~h}$ in the absence of thiamine (-T) to derepress $\mathrm{HO}$ endonuclease expression from the REP81X-HO plasmid. The percentage of colonies undergoing NHEJ/SCC ( $\mathrm{arg}^{+}$ $\mathrm{Hyg}^{\mathrm{R}}$ ade $^{+}$his $\left.^{+}\right)$, gene conversion $\left(\arg ^{+} \mathrm{Hyg}^{\mathrm{S}} \mathrm{ade}^{+}\right.$his $\left.{ }^{+}\right)$, minichromosome loss ( $\arg ^{-} \mathrm{Hyg}^{\mathrm{S}} \mathrm{ade}^{-} \mathrm{his}^{-}$) or LOH (arg ${ }^{+} \mathrm{Hyg}^{\mathrm{S}} \mathrm{ade}^{-}$his ${ }^{-}$) was calculated (see also Supplementary Fig. 1). To determine levels of break-induced minichromosome loss, background minichromosome loss at $48 \mathrm{~h}$ in blank vector assays was subtracted from break-induced minichromosome loss at $48 \mathrm{~h}$ in cells transformed with pREP81X-HO. More than 1,000 colonies were scored for each time point, and each experiment was performed three times using three independently derived strains for all mutants tested.

Fluorescent microscopy. Asynchronous cultures were treated with $+1-$ $5 \mu \mathrm{g} \mathrm{ml}{ }^{-1}$ bleomycin $\left(1 \mathrm{~h} 26^{\circ} \mathrm{C}\right)$, before being fixed in methanol. Samples were rehydrated and stained with 4',6-diamidino-2-phenylindole (DAPI) before examination using Zeiss Axioplan 2ie microscope, Hamamatsu Orca ER camera and micromanager software. For visualization of Rad11-GFP and Rad51-CFP foci, cells were irradiated with $50 \mathrm{~Gy}$ IR using a ${ }^{137} \mathrm{Cs}$ source with a dose rate of $2.8 \mathrm{~Gy}$ per minute, before being fixed and visualized as above.

Serial dilution assay. A dilution series for the indicated mutants was spotted onto YE6S plates and YE6S with the indicated concentration of MMS, bleomycin or bleocin. Plates were incubated at $32{ }^{\circ} \mathrm{C}$ for $2-3$ days before analysis.

lonizing radiation survival curve. Logarithmically growing cells were irradiated by using a ${ }^{60} \mathrm{Co}$ source at a dose rate of $31 \mathrm{~Gy} \mathrm{~min}^{-1}$. Irradiated and unirradiated cells were plated on YE6S and incubated at $32{ }^{\circ} \mathrm{C}$ for 4 days before colonies were counted.

Survival analysis. Exponential cultures were obtained in liquid YE6S medium inoculated with a single colony picked from a freshly streaked (YE6S) stock plate and grown overnight at $32^{\circ} \mathrm{C}$ with vigorous shaking. Cells were counted microscopically and only cultures with between $2 \times 10^{7}$ and $4 \times 10^{7}$ cells ml $^{-1}$ were used. Cells were resuspended in YE6S at a density of $2 \times 10^{7} \mathrm{cells} \mathrm{ml}^{-1}$, and serial dilutions were made and $\sim 200$ cells were plated on YE6S plates with the indicated dose of bleocin as well as an untreated control. Plates were incubated for 3 days at $32{ }^{\circ} \mathrm{C}$ and then scored.

Quantitative chromatin immunoprecipitation (qChIP). Chromatin immunoprecipitation (ChIP) was performed as previously described ${ }^{54}$. In brief, cells were grown at $32{ }^{\circ} \mathrm{C}$ in Edinburgh Minimal Medium (EMM) supplemented with $0.25 \mathrm{mg} / \mathrm{ml}$ uracil, to induce Purg1. One hundred millilitres of cells at O.D. ${ }_{595}=0.4$ were fixed in $1 \%$ formaldehyde (Sigma F8755-25ML) for $20 \mathrm{~min}$ at $24^{\circ} \mathrm{C}$ with shaking. The reaction was quenched by adding $125 \mathrm{mM}$ glycine for $5 \mathrm{~min}$. Cells were lysed using a bead beater (Biospec Products), and cell lysates were sonicated in a Bioruptor (Diagenode) (15 min, $30 \mathrm{~s}$ On and $30 \mathrm{~s}$ Off at 'High' (200 W) position). For all ChIPs, $30 \mu$ l Protein G Dynabeads (Life Technologies) were used 
along with $3.5 \mu \mathrm{l}$ ActiveMotif anti-H3K36me3 antibody (cat\# 61101) or $1.5 \mu \mathrm{l}$ antimyc 9B11 (Cell Signaling), as appropriate. ChIPs were analysed by real-time PCR using Lightcycler 480 SYBR Green (Roche) with primers specific to the indicated regions. All ChIP enrichments were calculated as \% DNA immunoprecipitated at the locus of interest (relative to the corresponding input samples) and normalized to \% DNA immunoprecipitated at the act1 or fbp1 locus. Data averaged over at least two biological replicates are shown. Error bars represent s.d. values from at least two biological replicates. The primers used for $\mathrm{qPCR}$ analysis are listed in Supplementary Table 3.

Micrococcal nuclease digestion of chromatin. One-hundred milliliters of cells were grown to mid-log phase in YE5S at $30^{\circ} \mathrm{C}$, treated with $3 \mu \mathrm{g} \mathrm{ml}^{-1}$ bleocin for $1 \mathrm{~h}$, crosslinked with a final concentration of $1 \%$ formaldehyde (Sigma F8775) for $20 \mathrm{~min}$ at $30^{\circ} \mathrm{C}$ and quenched by the addition of glycine to $125 \mathrm{mM}$. Cells were washed with CES buffer ( $50 \mathrm{mM}$ citric acid/50 $\mathrm{mM} \mathrm{Na}_{2} \mathrm{HPO}_{4}$ (pH5.6), $40 \mathrm{mM}$ EDTA (pH 8.0), 1.2 M sorbitol, $10 \mathrm{mM} \beta$-mercaptoethanol) and resuspended in $500 \mu \mathrm{l} \mathrm{CES} \mathrm{buffer} \mathrm{with} 0.5 \mathrm{mg}$ Zymolyase-100T. Cells were spheroplasted by gentle shaking at $30^{\circ} \mathrm{C}$ for up to $45 \mathrm{~min}$, washed with ice cold $1.2 \mathrm{M}$ sorbitol and resuspended in $800 \mu \mathrm{l} \mathrm{NP}$-S buffer $\left(1.2 \mathrm{M}\right.$ sorbitol, $10 \mathrm{mM} \mathrm{CaCl}_{2}, 100 \mathrm{mM} \mathrm{NaCl}$, $1 \mathrm{mM}$ EDTA (pH 8.0), $14 \mathrm{mM} \beta$-mercaptoethanol, $50 \mathrm{mM}$ Tris- $\mathrm{HCl}$ (pH 8.0), $0.075 \%$ NP-40, $5 \mathrm{mM}$ spermidine, $0.1 \mathrm{mM}$ PMSF, $1 \%$ Sigma protease inhibitor cocktail (Sigma P8215)). Spheroplasts were divided into four $200 \mu \mathrm{l}$ aliquots, each mixed with $300 \mu \mathrm{l}$ of NP-S buffer. MNase was added at the indicated concentrations, and samples were digested for $10 \mathrm{~min}$ at $37^{\circ} \mathrm{C}$. MNase activity was terminated by the addition of EDTA ( $\mathrm{pH} 8.0$ ) and SDS to the final concentrations of $50 \mathrm{mM}$ and $0.2 \%$, respectively. Samples were incubated at $65^{\circ} \mathrm{C}$ overnight with $0.2 \mathrm{mg} \mathrm{ml}^{-1}$ proteinase $\mathrm{K}$ and $10 \mu \mathrm{g}$ RNase A. DNA was subsequently purified by phenol:chloroform extraction followed by ethanol precipitation.

Plasmid rejoining assay. The plasmid rejoining assay was performed as previously described ${ }^{55}$. In brief, the cohesive-ended substrates for the NHEJ assay were prepared by excision of a $\sim 500 \mathrm{bp}$ PstI fragment or $\sim 700 \mathrm{bp}$ PvuII fragment from PS (p100) or a $\sim 540 \mathrm{bp} E c o R I$ fragment from PI (p101) followed by gel purification of the remaining linear vector. Logarithmically, growing cells $(20 \mathrm{ml}$ of OD595 0.5) were transformed with $1 \mu \mathrm{g}$ of undigested control plasmid pAL19 or linear DNA using the lithium acetate method. As the plasmids contain a LEU2 marker, NHEJ frequency was calculated as the percentage of leu ${ }^{+}$colonies arising from cells transformed with linear plasmid over those transformed with undigested DNA. At least three experiments were performed for each strain, and the average percentage rejoining calculated.

Microarray analysis. Microarray analysis was performed as previously described $^{56}$. In brief, Alexa 555- or 647-labelled cDNA was produced from the RNA, using a Superscript direct cDNA labelling system (Invitrogen) and Alexa 555 and 647 dUTP label mix. The cDNA was then purified using an Invitrogen PureLink PCR Purification system. The cDNA was hybridized to the array using a Gene Expression Hybridization kit (Agilent). The array was an Agilent custom-designed array containing 60-mer oligonucleotides synthesized in situ on the array and contained $4 \times 44,000$ probes. Following hybridization for at least $17 \mathrm{~h}$, the array was washed using a Gene Expression Wash Buffer kit (Agilent) and scanned in an Agilent Array Scanner. The microarray signal was extracted using GenePix.

Analysis of cell cycle-regulated histone modifications. The wild-type yeast strain was arrested in G1 using nitrogen starvation ${ }^{57}$ and released from the block. Samples were taken over a 5-h time course. The samples then underwent the histone acid-extraction method to purify histones for analysis by western blotting as previously described ${ }^{58}$. In brief, one litre of cells were collected by centrifugation. The cell pellet was resuspended in spheroplasting buffer $(1.2 \mathrm{M}$ Sorbitol, $20 \mathrm{mM}$ Hepes pH 7.4, $1 \mathrm{mM}$ PMSF, $0.5 \mu \mathrm{g} \mathrm{ml}^{-1}$ Leupeptin, $0.7 \mu \mathrm{g} \mathrm{ml}^{-1}$ Pepstatin) containing $10 \mathrm{mM}$ dithiothreitol (DTT) and $2 \mathrm{mg} \mathrm{ml}^{-1}$ Zymolyase 20-T (MP Biomedicals) and then incubated in a $32{ }^{\circ} \mathrm{C}$ water bath until $90 \%$ of cells had lost their cell walls. Cells were centrifuged and resuspended in Nuclei Isolation Buffer (0.25 M Sucrose, $60 \mathrm{mM} \mathrm{KCl}, 14 \mathrm{mM} \mathrm{NaCl}, 5 \mathrm{mM} \mathrm{MgCl} 2,1 \mathrm{mM} \mathrm{CaCl} 2,15 \mathrm{mM}$ MES, pH 6.6, $0.8 \%$ Triton X-100, $0.7 \mu \mathrm{g} \mathrm{ml}{ }^{-1}$ Pepstatin, $1 \mathrm{mM} \mathrm{PMSF}$,

$0.5 \mathrm{\mu g} \mathrm{ml}^{-1}$ Leupeptin) on ice water for $20 \mathrm{~min}$. After spinning and washing, most of the chromatin was in the pellet. Histones were extracted by resuspending the pellet in $10 \mathrm{ml}$ of cold $0.4 \mathrm{~N} \mathrm{H}_{2} \mathrm{SO}_{4}$. Protein extracts were made by TCA extraction and analysed by western blotting as previous described ${ }^{58}$. H3 tri-methyl lysine 36 ( $\alpha \mathrm{H} 3 \mathrm{~K} 36 \mathrm{me} 3$ ) (Abcam 9050), H3 di-methy lysine 36 ( $\alpha \mathrm{H} 3 \mathrm{~K} 36 \mathrm{me} 2)$ (Abcam 9049), $\mathrm{H} 3$ mono-methyl lysine 36 ( $\alpha \mathrm{H} 3 \mathrm{~K} 36 \mathrm{mel}$ ) (Abcam 9048) and $\mathrm{H} 3$ lysine 36 acetylation (Active Motif 39379) were used at a dilution of 1:1,000. An antibody directed against H3 (Abcam1791) was used as a loading control. Anti-rabbit horseradish peroxidase-conjugated secondary antibodies (Amersham Bioscience) were used at a dilution of 1:10,000. For clarity, western blots were cropped to show the band of interest in the main figures. However, corresponding uncropped scans can be found in Supplementary Fig. 10 and Supplementary Fig. 11.

\section{References}

1. Symington, L. S. \& Gautier, J. Double-strand break end resection and repair pathway choice. Annu. Rev. Genet. 45, 247-271 (2011).

2. Lieber, M. R. The mechanism of double-strand DNA break repair by the nonhomologous DNA end-joining pathway. Annu. Rev. Biochem. 79, 181-211 (2010).

3. Heyer, W. D., Ehmsen, K. T. \& Liu, J. Regulation of homologous recombination in eukaryotes. Annu. Rev. Genet. 44, 113-139 (2010)

4. Aylon, Y., Liefshitz, B. \& Kupiec, M. The CDK regulates repair of double-strand breaks by homologous recombination during the cell cycle. EMBO J. 23, 4868-4875 (2004).

5. Ira, G. et al. DNA end resection, homologous recombination and DNA damage checkpoint activation require CDK1. Nature 431, 1011-1017 (2004).

6. Ferreira, M. G. \& Cooper, J. P. Two modes of DNA double-strand break repair are reciprocally regulated through the fission yeast cell cycle. Genes Dev. 18, 2249-2254 (2004).

7. Wagner, E. J. \& Carpenter, P. B. Understanding the language of Lys36 methylation at histone H3. Nat. Rev. Mol. Cell Biol. 13, 115-126 (2012).

8. Strahl, B. D. et al. Set2 is a nucleosomal histone H3-selective methyltransferase that mediates transcriptional repression. Mol. Cell Biol. 22, 1298-1306 (2002)

9. Edmunds, J. W., Mahadevan, L. C. \& Clayton, A. L. Dynamic histone H3 methylation during gene induction: $\mathrm{HYPB} / \mathrm{Setd} 2$ mediates all $\mathrm{H} 3 \mathrm{~K} 36$ trimethylation. EMBO J. 27, 406-420 (2008).

10. Al Sarakbi, W. et al. The mRNA expression of SETD2 in human breast cancer correlation with clinico-pathological parameters. BMC Cancer 9, 290 (2009).

11. Dalgliesh, G. L. et al. Systematic sequencing of renal carcinoma reveals inactivation of histone modifying genes. Nature 463, 360-363 (2010).

12. Newbold, R. F. \& Mokbel, K. Evidence for a tumour suppressor function of SETD2 in human breast cancer: a new hypothesis. Anticancer Res. 30, 3309-3311 (2010).

13. Zhang, J. et al. The genetic basis of early T-cell precursor acute lymphoblastic leukaemia. Nature 481, 157-163 (2012).

14. Fontebasso, A. M. et al. Mutations in SETD2 and genes affecting histone H3K36 methylation target hemispheric high-grade gliomas. Acta Neuropathol. 125, 659-669 (2013).

15. Jazayeri, A., McAinsh, A. D. \& Jackson, S. P. Saccharomyces cerevisiae Sin3p facilitates DNA double-strand break repair. Proc. Natl Acad. Sci. USA 101, 1644-1649 (2004).

16. Merker, J. D. et al. The histone methylase Set2p and the histone deacetylase Rpd3p repress meiotic recombination at the HIS4 meiotic recombination hotspot in Saccharomyces cerevisiae. DNA. Repair 7, 1298-1308 (2008).

17. Fnu, S. et al. Methylation of histone H3 lysine 36 enhances DNA repair by nonhomologous end-joining. Proc. Natl Acad. Sci. USA 108, 540-545 (2011).

18. Daugaard, M. et al. LEDGF (p75) promotes DNA-end resection and homologous recombination. Nat. Struct. Mol. Biol. 19, 803-810 (2012).

19. Morris, S. A. et al. Identification of histone H3 lysine 36 acetylation as a highly conserved histone modification. J. Biol. Chem. 282, 7632-7640 (2007).

20. Daniel, J. A. \& Grant, P. A. Multi-tasking on chromatin with the SAGA coactivator complexes. Mutat. Res. 618, 135-148 (2007).

21. Barlev, N. A. et al. Repression of GCN5 histone acetyltransferase activity via bromodomain-mediated binding and phosphorylation by the Ku-DNAdependent protein kinase complex. Mol. Cell Biol. 18, 1349-1358 (1998).

22. Tamburini, B. A. \& Tyler, J. K. Localized histone acetylation and deacetylation triggered by the homologous recombination pathway of double-strand DNA repair. Mol. Cell Biol. 25, 4903-4913 (2005).

23. Oishi, H. et al. An hGCN5/TRRAP histone acetyltransferase complex coactivates BRCA1 transactivation function through histone modification. J. Biol Chem. 281, 20-26 (2006).

24. Lee, H. S., Park, J. H., Kim, S. J., Kwon, S. J. \& Kwon, J. A cooperative activation loop among SWI/SNF, gamma-H2AX and $\mathrm{H} 3$ acetylation for DNA doublestrand break repair. EMBO J. 29, 1434-1445 (2010).

25. Lee, S. E. et al. Saccharomyces Ku70, mre11/rad50 and RPA proteins regulate adaptation to G2/M arrest after DNA damage. Cell 94, 399-409 (1998).

26. Pierce, A. J., Hu, P., Han, M., Ellis, N. \& Jasin, M. Ku DNA end-binding protein modulates homologous repair of double-strand breaks in mammalian cells. Genes Dev. 15, 3237-3242 (2001).

27. Rea, S. et al. Regulation of chromatin structure by site-specific histone H3 methyltransferases. Nature 406, 593-599 (2000).

28. Morris, S. A. et al. Histone H3 K36 methylation is associated with transcription elongation in Schizosaccharomyces pombe. Eukaryot. Cell 4, 1446-1454 (2005).

29. Tinline-Purvis, H. et al. Failed gene conversion leads to extensive end processing and chromosomal rearrangements in fission yeast. EMBO J. 28, 3400-3412 (2009).

30. Keogh, M. C. et al. Cotranscriptional set2 methylation of histone H3 lysine 36 recruits a repressive Rpd3 complex. Cell 123, 593-605 (2005).

31. Nakayama, J. et al. Alp13, an MRG family protein, is a component of fission yeast Clr6 histone deacetylase required for genomic integrity. EMBO J. 22, 2776-2787 (2003). 
32. Nicolas, E. et al. Distinct roles of HDAC complexes in promoter silencing, antisense suppression and DNA damage protection. Nat. Struct. Mol. Biol. 14, 372-380 (2007).

33. Carneiro, T. et al. Telomeres avoid end detection by severing the checkpoint signal transduction pathway. Nature 467, 228-232 (2010).

34. Barlow, J. H., Lisby, M. \& Rothstein, R. Differential regulation of the cellular response to DNA double-strand breaks in G1. Mol. Cell 30, 73-85 (2008).

35. Wang, Y. et al. Histone $\mathrm{H} 3$ lysine 14 acetylation is required for activation of a DNA damage checkpoint in fission yeast. J. Biol. Chem. 287, 4386-4393 (2012).

36. Johnsson, A., Xue-Franzen, Y., Lundin, M. \& Wright, A. P. Stress-specific role of fission yeast Gcn5 histone acetyltransferase in programming a subset of stress response genes. Eukaryot. Cell 5, 1337-1346 (2006).

37. Benson, L. J. et al. Properties of the type B histone acetyltransferase Hat1: H4 tail interaction, site preference, and involvement in DNA repair. J. Biol. Chem. 282, 836-842 (2007).

38. Mitchell, L. et al. Functional dissection of the NuA4 histone acetyltransferase reveals its role as a genetic hub and that Eafl is essential for complex integrity. Mol. Cell Biol. 28, 2244-2256 (2008).

39. Qin, S. \& Parthun, M. R. Histone H3 and the histone acetyltransferase Hatlp contribute to DNA double-strand break repair. Mol. Cell Biol. 22, 8353-8365 (2002).

40. Shim, E. Y., Ma, J. L., Oum, J. H., Yanez, Y. \& Lee, S. E. The yeast chromatin remodeler RSC complex facilitates end joining repair of DNA double-strand breaks. Mol. Cell Biol. 25, 3934-3944 (2005).

41. Shim, E. Y. et al. RSC mobilizes nucleosomes to improve accessibility of repair machinery to the damaged chromatin. Mol. Cell Biol. 27, 1602-1613 (2007).

42. Blier, P. R., Griffith, A. J., Craft, J. \& Hardin, J. A. Binding of Ku protein to DNA. Measurement of affinity for ends and demonstration of binding to nicks. J. Biol. Chem. 268, 7594-7601 (1993).

43. Venkatesh, S. et al. Set2 methylation of histone H3 lysine 36 suppresses histone exchange on transcribed genes. Nature 489, 452-455 (2012).

44. Smolle, M. et al. Chromatin remodelers Isw1 and Chd1 maintain chromatin structure during transcription by preventing histone exchange. Nat. Struct. Mol. Biol. 19, 884-892 (2012).

45. Adkins, N. L., Niu, H., Sung, P. \& Peterson, C. L. Nucleosome dynamics regulates DNA processing. Nat. Struct. Mol. Biol. 20, 836-842 (2013).

46. Bird, A. W. et al. Acetylation of histone H4 by Esal is required for DNA double-strand break repair. Nature 419, 411-415 (2002)

47. Bennett, G., Papamichos-Chronakis, M. \& Peterson, C. L. DNA repair choice defines a common pathway for recruitment of chromatin regulators. Nat. Commun. 4, 2084 (2013).

48. Jha, D. K. \& Strahl, B. D. An RNA polymerase II-coupled function for histone H3K36 methylation in checkpoint activation and DSB repair. Nat. Commun. 5, 3965 doi:10.1038/ncomms4965 (2014).

49. Aymard, F. et al. Transcriptionally active chromatin recruits homologous recombination at DNA double-strand breaks. Nat. Struct. Mol. Biol. 21, 366-374 (2014).

50. Pfister, S. X. et al. ETD2-dependent histone H3K36 trimethylation is required for homologous recombination repair and genome stability. Cell Reports (in press, 2014).

51. Faucher, D. \& Wellinger, R. J. Methylated H3K4, a transcription-associated histone modification, is involved in the DNA damage response pathway. PLoS Genet. 6 pii e1001082 (2010).

52. Li, F. et al. The Histone Mark H3K36me3 Regulates Human DNA Mismatch Repair through Its Interaction with MutSalpha. Cell 153, 590-600 (2013).

53. Moreno, S., Klar, A. \& Nurse, P. Molecular genetic analysis of fission yeast Schizosaccharomyces pombe. Methods Enzymol. 194, 795-823 (1991).
54. Subramanian, L. \& Nakamura, T. M. A kinase-independent role for the Rad3(ATR)-Rad26(ATRIP) complex in recruitment of Tel1(ATM) to telomeres in fission yeast. PLoS Genet. 6, e1000839 (2010).

55. Manolis, K. G. et al. Novel functional requirements for non-homologous DNA end joining in Schizosaccharomyces pombe. EMBO J. 20, 210-221 (2001).

56. Rallis, C., Codlin, S. \& Bahler, J. TORC1 signaling inhibition by rapamycin and caffeine affect lifespan, global gene expression, and cell proliferation of fission yeast. Aging Cell 12, 563-573 (2013)

57. Nurse, P. \& Bissett, Y. Gene required in G1 for commitment to cell cycle and in G2 for control of mitosis in fission yeast. Nature 292, 558-560 (1981).

58. Shechter, D., Dormann, H. L., Allis, C. D. \& Hake, S. B. Extraction, purification and analysis of histones. Nat. Protoc. 2, 1445-1457 (2007).

59. Watson, A. T., Werler, P. \& Carr, A. M. Regulation of gene expression at the fission yeast Schizosaccharomyces pombe urg1 locus. Gene 484, 75-85 (2011).

\section{Acknowledgements}

We thank the Kearsey lab for the use of their fluorescence microscope and the Carr lab for strains and reagents. We thank Brian Strahl (UNC-Chapel Hill) for communicating results before publication. C.C.P., R.S.D., S.S., E.J.B., C.W., L.H., E.B. and T.C.H. were supported by the Medical Research Council (R06538; R19583); L.S. was supported by an EC FP7 Marie Curie International Incoming Fellowship (PIIF-GA-2010-275280) and an EMBO Long Term Fellowship (ALTF 1491-2010). The Wellcome Trust supported the work of RCA (095021 and 065061) and the Wellcome Trust Centre for Cell Biology (092076); S.C. and J.B. were supported by a Wellcome Trust Senior Investigator Award (095598/Z/11/Z). C.S. and S.W. were supported by the Medical Research Council and the National Institute for Health Research (NHIR) Newcastle Biomedical Research Centre based at Newcastle upon Tyne Hospitals NHS Foundation Trust and Newcastle University. The views expressed are those of the authors and not necessarily those of the NHS, NHIR or the Department of Health.

\section{Author contributions}

C.C.P., R.S.D., L.S., S.S., E.J.B., C.W. and L.H. designed and performed experiments with input from T.C.H. in strain engineering. T.C.H. supervised the work and wrote the manuscript with input from all authors. Experiments in Fig. 1 were performed by C.C.P., R.S.D. and C.W.; in Fig. 2 were performed by C.C.P., S.S., R.S.D. and L.S.; in Fig. 3 by C.C.P., R.S.D. and E.J.B.; in Fig. 4 by C.C.P., R.S.D. and C.W. with strain engineering from R.A.; in Fig. 5 were designed by S.W. and performed by C.G.; in Fig. 6 were performed by C.C.P. S.C. and J.B. performed experiments presented in Supplementary Fig. 3.

\section{Additional information}

Accession codes: The microarray data have been deposited with ArrayExpress (https://www.ebi.ac.uk/arrayexpress/) under accession code E-MTAB-2549.

Supplementary Information accompanies this paper at http://www.nature.com/ naturecommunications

Competing financial interests: The authors declare no competing financial interests

Reprints and permission information is available online at http://npg.nature.com/ reprintsandpermissions/

How to cite this article: Pai, C.-C. et al. A histone H3K36 chromatin switch coordinates DNA double-strand break repair pathway choice. Nat. Commun. 5:4091 doi: 10.1038/ncomms5091 (2014). 